\title{
Ellipsometric study of thermally induced redistribution and crystallization of Ge in Ge:SiO 2 mixture layers
}

\author{
Vesna Janicki ${ }^{*}$, Jordi Sancho Parramon, Hrvoje Zorc, Krešimir Salamon, Maja Buljan, Nikola \\ Radić, Uroš Desnica
}

Ruđer Bošković Institute, Bijenička c. 54, 10000 Zagreb, Croatia

\begin{abstract}
Mixture layers of $\mathrm{Ge}: \mathrm{SiO}_{2}$ of 40:60\% mol respectively, have been prepared by co-sputtering. The thermally induced change of optical properties of the layers was studied by variable angle spectroscopic ellipsometry. The mixture was modelled as an unknown material with optical constants described by multiple oscillators. The optical parameters determined from ellipsometric measurements can be well correlated with structural changes in the mixture. The results indicate that $\mathrm{Ge}$ in the mixture deposited or annealed up to $600^{\circ} \mathrm{C}$ is in an amorphous state and it redistributes with increase of temperature, changing refractive index through the layer. The crystallization starts between 600 and $650^{\circ} \mathrm{C}$, at first next to the substrate. Crystallites size grows with temperature. Results were compared with findings of grazing incidence wide angle x-ray scattering measurements and a good agreement was found. Ellipsometry has been shown to be an appropriate non-invasive technique for characterization of this kind of layers.
\end{abstract}

*Corresponding author: janicki@ irb.hr, tel. +385 1 4571247, fax. + 38514680108

\section{Introduction}

Ge quantum dots embedded in a transparent matrix are interesting for their strong photo- and electro-luminescence, third-order optical nonlinearities and tailorable absorption. These properties and their tunability depend on nanoparticle size, size distribution and their correlation [1]. Therefore, it is of key importance to control the size, shape and arrangement of Ge nanoparticles in the matrix. The structural studies of $\mathrm{Ge}$ nanoparticle formation in $\mathrm{SiO}_{2}$ matrix depending on substrate $\left(\mathrm{T}_{\mathrm{s}}\right)$ and annealing $\left(\mathrm{T}_{\mathrm{a}}\right)$ temperature have been done for a mixture of $\mathrm{Ge}$ and $\mathrm{SiO}_{2}$ as single layers [2] and as multilayers where mixture layers are separated by $\mathrm{SiO}_{2}[3,4]$.

Modelling of optical properties of Ge quantum dots in a dielectric matrix has been done in different ways using effective medium approximation (EMA) [5]. However, using optical constants of bulk Ge in EMA for characterization of a mixture containing Ge quantum dots can fail because the optical constants of dots can be quite different than those of material as bulk. Solutions proposed to solve this problem are finding volume fraction and optical constants of the quantum dots by direct inversion [6,7], using dispersion model [8] or using splines [9]. These approaches were applied always to the homogeneous thin films, where Ge was homogeneously distributed through the film. However, for the samples studied here the 
situation is more complex, as they show inhomogeneity (change of refractive index with thickness of the layer) due to Ge depth distribution, but also Ge appears amorphous or crystalline, as will be seen.

The samples studied here contain Ge clusters of different sizes in $\mathrm{SiO}_{2}$ matrix. This mixture of materials could be even on the level of atoms, meaning that the compounds could be present on atomic basis, with no clusters formed. Also, these clusters can be either amorphous or crystalline. The size of crystallites is expected to change with $T_{s}$ and $T_{a}$ higher than the temperature of crystallization onset. Therefore, using EMA with the optical constants of bulk Ge results in poor data fits and failure to describe optical properties of the samples. The other problem of using EMA in this case is that the mixture could evolve from an atomic level mixture to a mixture with well defined phases where $\mathrm{Ge}$ is in the shape of clusters. Thus also the type of used EMA should evolve from Lorentz-Lorenz to MaxwellGarnet or Bruggeman [10, 11].

To avoid these problems another approach was used. The mixture in the layer was treated as an unknown material, which will be called material $\mathrm{M}$ here. The optical constants of material $\mathbf{M}$ were modelled using multiple oscillators: Gauss oscillators [12] appeared to be well-suited to describe the optical constants around critical points while the CodyLorentz model [13] was used to take into account possible Urbach tail absorption and absorption around the band-gap region. This unknown material $\mathrm{M}$ contains $\mathrm{SiO}_{2}$ and $\mathrm{Ge}$ in the shape of atoms or clusters of different size and crystallinity. Therefore, the parameters in the oscillator model will change accordingly from sample to sample. Although this approach will not give optical constants of $\mathrm{Ge}$ in the mixture, it evidences onset of crystallization and cluster growth, allowing using ellipsometry for obtaining structural information of the complex samples.

\section{Experimental details}

The samples were prepared by magnetron sputtering co-deposition from $\mathrm{Ge}$ and $\mathrm{SiO}_{2}$ targets in DC (14 W) and RF (250 W) operated magnetrons, respectively. The nominal mixture in the layers was $40 \% \mathrm{~mol} \mathrm{Ge}$ and $60 \% \mathrm{~mol} \mathrm{SiO}_{2}$. This corresponds to approximate volume fraction $\left(f_{v}\right)$ of $\mathrm{Ge}$ of $30 \%$. All the layers were deposited onto $<111>$ Si substrates that were previously etched to remove native $\mathrm{SiO}_{2}$ layer.

The layers are deposited on substrates pre-heated to different temperatures. The substrate temperature $\left(\mathrm{T}_{\mathrm{s}}\right)$ ranged from room temperature $(\mathrm{RT})$ to $700^{\circ} \mathrm{C}$. Some of the samples were subsequently thermally annealed in vacuum for one hour to the annealing temperature ( $\mathrm{T}_{\mathrm{a}}$ ) up to $900^{\circ} \mathrm{C}$. The samples in the figures are named by the temperature of substrate and annealing as $\mathrm{T}_{\mathrm{s}}+\mathrm{T}_{\mathrm{a}}$. For example, $300^{\circ} \mathrm{C}+600^{\circ} \mathrm{C}$ is the sample deposited at substrate heated at $300^{\circ} \mathrm{C}$ and annealed at $600^{\circ} \mathrm{C}$. When only one number is present, it corresponds to the substrate temperature.

Ellipsometric measurements of $\boldsymbol{\psi}$ and $\Delta$ functions were carried out by Woollam VASE ellipsometer in the wavelength range $270-2200 \mathrm{~nm}(0.564-4.593 \mathrm{eV})$ each $10 \mathrm{~nm}$, at 
three angles of incidence: $65^{\circ}, 70^{\circ}$ and $75^{\circ}$. The analysis of experimental data was done with WVASE32 software.

Grazing incidence wide angle x-ray scattering (GIWAXS) measurements were performed under fixed incidence angle $\alpha_{\mathrm{i}}=0.5^{\circ}$ being large enough to allow the X-rays to penetrate throughout the whole film and small enough to reduce the substrate contribution. The GIWAXS spectra were acquired using $\mathrm{Cu}$ radiation $(\lambda=0.154 \mathrm{~nm})$ and a curved position sensitive detector allowing the pattern to be collected in the $2 \theta$ range $20^{\circ}-65^{\circ}$.

\section{Modelling}

The optical properties of the studied Ge-Si mixture in the layers were related to the measured data by a model of the mixture. Some of the parameters that define the model are thickness of the layer, nonuniformity, degree of inhomogeneity, parameters of dispersion model, etc. The values of the parameters were optimized in order to obtain the best fit of the simulated optical properties of the model to the measured ellipsometric data.

As the first step, to obtain approximate initial thickness and optical constants of the layer, which would be later used for setting the initial oscillator parameters values, the data range was limited to the wavelengths free of Ge absorption $(\geq 500 \mathrm{~nm})$ and a simple Cauchy refractive index model was used to model initial optical constants. Thus, approximate thickness of the layers was obtained.

The second step was to fix thickness, employ the whole range of measured data and obtain a complex refractive index from wavelength by wavelength analysis. Thus obtained complex refractive index data were used for choosing a proper dispersion model to represent the average optical constants of mixture material M.

Introduction of surface roughness, modelled as Bruggeman mixture of $50 \% f_{v}$ air and $50 \% f_{v}$ of material $\mathrm{M}$, gave no significant improvement to the quality of the fit. Depolarization maxima (measured together with ellipsometric $\psi$ and $\Delta$ functions) match with $\psi$ and $\Delta$ maxima indicating thickness nonuniformity. Indeed, when nonuniformity was included in the modelling ( $2-3.5 \%$ of nonuniformity), the quality of the fit improved significantly. Afterwards, the thickness of the coating was also refined.

Finally, refractive index profile, i.e. inhomogeneity, was introduced in the model gradually, with increasing complexity of the refractive index profile. Inhomogeneity was introduced by dividing the layer of total thickness $d_{T}$ into several sublayers. The sublayer with the highest refractive index $n$ was treated as $100 \% f_{v}$ of material M. The optical constants of sublayers with lower $n$ were treated as a Bruggeman EMA mixture of $\mathrm{M}$ and $\mathrm{SiO}_{2}$. Introduction of inhomogeneity improved the quality of the fit significantly. It must be highlighted that material $\mathrm{M}$ is defined as containing some $\mathrm{SiO}_{2}$ besides $\mathrm{Ge}$, but it is the sublayer with lowest content of $\mathrm{SiO}_{2}$ and highest content of $\mathrm{Ge}$ in the model. This was done to avoid the problem of applying the type of EMA appropriate to the growing size of $\mathrm{Ge}$ 
clusters. However, in the case of inhomogeneous layer this approach still has a limitation. Depending on Ge concentration through the layer, Ge clusters may appear in different sizes [14], having different optical constants, that will not be taken into account by material $\mathrm{M}$ parameters. The present approach gives only a kind of average contribution of Ge to optical constants of the material $\mathrm{M}$ through the layer. However, good quality fittings of the measurements of the studied samples could be obtained only by using this kind of complex model.

\section{Results}

In this section analysis of the fits quality is presented, same as the evolution of the parameters of the model with the increase of the temperature that is obtained from the fitting of the experimental data.

To check the quality of the obtained fits, Fig. 1 shows measured ellipsometric functions $\psi$ and $\Delta$ data and the model simulated data corresponding to the best fit for the sample deposited on substrate heated to $300^{\circ} \mathrm{C}$ and later annealed at $600^{\circ} \mathrm{C}$. This sample has the worst final fit. Generally, the fits of all the samples have very good quality, with discrepancy between model and experimental data comparable to the experimental error. In fact, an inspection of the graphs, by eye only, barely can distinguish the best from the poorer fits. To get a more quantitative idea about the quality, Fig. 2 shows the difference of fit to the measured values of the best and worst final fit obtained for the studied samples.

Total thickness of the layer is reducing as $\mathrm{T}_{\mathrm{s}}$ and $\mathrm{T}_{\mathrm{a}}$ are increasing (Fig. 3). Refractive

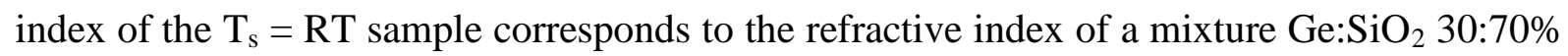
$f_{v}$. This composition value is extracted from effective medium approximation. Similar composition values are obtained regardless the used effective medium approximation, since at the corresponding wavelength the composing materials are weakly absorbing and different effective medium approximations lead to comparable results [15]. The layer has a positive inhomogeneity (increase of refractive index with thickness of the layer) of $8 \%$. The refractive index profile $(n(d))$ of the studied samples changes with $\mathrm{T}_{\mathrm{s}}$ and $\mathrm{T}_{\mathrm{a}}$ (Fig. 4). The profiles show drop of $n$ towards the surface of the layer.

In the same time, the average refractive index $\left(n_{\text {ave }}\right)$ of the layer, as well as maximal $n$ in the layer, decreases (Fig. 5). This trend develops until $650^{\circ} \mathrm{C}$ (for the sample $\mathrm{T}_{\mathrm{s}}=650^{\circ} \mathrm{C}$ and the sample $\mathrm{T}_{\mathrm{s}}=300^{\circ} \mathrm{C} / \mathrm{T}_{\mathrm{a}}=650^{\circ} \mathrm{C}$ ) when the layers present constant refractive index profiles having the lowest $n_{\text {ave }}$ at $2.48 \mathrm{eV}$. The extinction coefficient $k$ at this energy is $\sim 10^{-3}$. The further increase of $\mathrm{T}_{\mathrm{a}}\left(700^{\circ} \mathrm{C}-900^{\circ} \mathrm{C}\right)$ increases $n$ in the part of the layer next to the substrate. For the highest $\mathrm{T}_{\mathrm{a}}=900^{\circ} \mathrm{C}$, refractive index in the first half of the layer thickness (from the substrate) recovers $80 \%$ in magnitude, compared with RT sample. The change in the trend of refractive index evolution coincides with the crystallization onset seen by GIWAXS (Fig. 6). This indicates that Ge is distributed throughout the layer in different ways before and after crystallization. 
Real and imaginary parts of dielectric function $\left(\varepsilon_{1}, \varepsilon_{2}\right.$, respectively) decrease with temperature until $650^{\circ} \mathrm{C}$ (Fig. 7 and Fig. 8). At $\mathrm{T}_{\mathrm{s}}$ or $\mathrm{T}_{\mathrm{a}} \leq 600^{\circ} \mathrm{C} \varepsilon_{1}$ shows a broad peak around $2.4 \mathrm{eV}$, which can be associated to amorphous $\mathrm{Ge}$ [16]. Further increase of $\mathrm{T}_{\mathrm{a}}$ causes increase of $\varepsilon_{1}$ and $\varepsilon_{2}$ and appearance of peaks that can be related to critical points (CPs) of crystalline Ge band structure $[17,18]$.

Absorption properties of material $\mathrm{M}$ are seen in imaginary part of dielectric function (Fig. 8). The RT sample, the same as those annealed or deposited at temperature $\leq 600^{\circ} \mathrm{C}$, has an $\varepsilon_{2}$ that presents a long Urbach tail that decreases in intensity with increase of $T_{\mathrm{s}}$ and $\mathrm{T}_{\mathrm{a}}$. Overall intensity of $\varepsilon_{2}$ of these samples is reducing with $\mathrm{T}_{\mathrm{s}}$ and $\mathrm{T}_{\mathrm{a}}$ in the studied range of energies. In addition to the tail, there is a broad peak around $3 \mathrm{eV}$ that coincides with the characteristic for amorphous Ge [16]. The samples annealed or deposited at $650^{\circ} \mathrm{C}$ show negligible absorption. At even higher temperatures, $\varepsilon_{2}$ increases, showing peaks related to CPs of crystalline Ge.

The positions of CPs in the band structure correspond to maxima in $\varepsilon_{2}$. They are determined by minima of the second derivative of $\varepsilon_{2}$ [7-9]. In this case it is proper to use $\varepsilon_{2}$ dispersion obtained from wavelength by wavelength analysis because otherwise CPs positions could be imposed by the used dispersion model. On the other hand, data extracted from wavelength by wavelength analysis are quite noisy, so they have to be smoothed first. $\boldsymbol{E}_{\boldsymbol{I}}$ peak is a CP that is related to transitions in the $\Lambda$ directions of the Brillouin zone [17]. The position of $E_{l}$ peak for various samples is presented in Table 1. It can be compared with the position of $E_{1}$ peak in bulk crystalline Ge, that is located at $2.1 \mathrm{eV}$. The shift of $E_{1}$ peak is related to the size of crystallites: the smaller the shift the bigger the clusters [19].

In Fig. 6 the net GIWAXS patterns are plotted after proper normalization and subtraction of the background ( $\mathrm{Si}$ substrate) intensity. Three peaks in the net GIWAXS patterns, marked as 111, 220 and 311, correspond to the randomly oriented crystallites of the Ge FCC structure. The peaks are rather diffuse for $\mathrm{T}_{\mathrm{a}}$ or $\mathrm{T}_{\mathrm{s}}<600^{\circ} \mathrm{C}$, while narrowing up with temperature increasing above $600^{\circ} \mathrm{C}$. Broad maximum at the position of the Ge 111 for $\mathrm{T}_{\mathrm{a}}$ or $\mathrm{T}_{\mathrm{s}}<600^{\circ} \mathrm{C}$ could be attributed to very small non-precipitated clusters of $\mathrm{Ge}$ atoms. Average crystallite size for each sample was estimated from Bragg peaks in Fig. 6, by using Scherrer's formula [20]. The estimated crystallite size $D$ increases with temperature: from $4 \mathrm{~nm}$ at $\mathrm{T}_{\mathrm{a}}$ or $\mathrm{T}_{\mathrm{s}}=600^{\circ} \mathrm{C}$ to $8 \mathrm{~nm}$ at $\mathrm{T}_{\mathrm{a}}=900^{\circ} \mathrm{C}$. For the samples annealed at 800 and $900^{\circ} \mathrm{C}$, the integrated intensities of Bragg's peaks are damped, but their FWHM is also slightly diminished. This indicates the formation of larger Ge crystallites, but in lower volume fraction. It can be attributed to the out diffusion of Ge atoms from the sample [3], that is more enhanced for $\mathrm{T}_{\mathrm{a}}=$ $900^{\circ} \mathrm{C}$. Fig. 9 shows electron density in the surface of the samples calculated from X-ray reflectance measurements. It is possible to see that electron density decreases with increase of substrate or annealing temperature. This is in accordance with a decrease of $n$ at the surface of the layer with temperature (see Fig. 4).

\section{Discussion}


The evolution of $n$, which is related with migration of Ge, changes the trend between 600 and $650^{\circ} \mathrm{C}$. This is also the temperature range where GIWAXS results show that crystallization of $\mathrm{Ge}: \mathrm{SiO}_{2}$ 40:60\% mol samples start. This indicates that Ge distributes in a different way depending upon wether clusters have crystallized or not. Ge deposited and annealed at temperatures below $650^{\circ} \mathrm{C}$ is amorphous. The Urbach tail of the samples, which is related to the measure of defects and ordering in the matrix [21], decreases with annealing at these low temperatures indicating relaxation of the matrix with redistribution of Ge. Atomic Ge in a dielectric matrix cannot show absorption properties as in bulk or clusters. The initial decrease of $\varepsilon_{2}$ with $\mathrm{T}_{\mathrm{s}}$ or $\mathrm{T}_{\mathrm{a}}$ could represent such "melting" of Ge clusters in the $\mathrm{SiO}_{2}$ matrix, reaching maximum of solubility between 600 and $650^{\circ} \mathrm{C}$, just at the beginning of crystallization when crystallites start to grow. This is evident by peak-like features in $\varepsilon_{2}$ related to CPs of crystalline Ge. Formation of Ge crystallites initiates near the substrate thus increasing $n$ in the first half of the layer. From Table 1 it is possible to see the trend of the $E_{l}$ shift, indicating particle growth as $\mathrm{T}_{\mathrm{a}}$ increases. However, due to the noise in dispersion data obtained from wavelength by wavelength analysis the errors in crystallite size estimates are too big, so no such estimations are presented here. Also, one has to keep in mind that $\varepsilon_{2}$ is related to material $\mathrm{M}$, not to Ge. However, results clearly show the proper trend of growth of Ge clusters.

\section{Conclusions}

Thermally induced optical properties change of co-sputtered $\mathrm{Ge}: \mathrm{SiO}_{2}$ mixture of nominal ratio 40:60\% mol respectively, has been studied by variable angle spectroscopic ellipsometry. The mixture has been deposited on pre-heated substrates and some of the samples have been annealed additionally, up to $900^{\circ} \mathrm{C}$.

It is found that $\mathrm{Ge}$ is present in the layers in the amorphous state for temperatures up to $600^{\circ} \mathrm{C}$. In this range of temperatures $\varepsilon_{2}$ is decreasing, indicating redistribution of Ge. Crystallization starts between 600 and $650^{\circ} \mathrm{C}$ and next to the substrate. The size of crystallites increases with temperature, which is accompanied by the shift in the position of critical points.

Treating the mixture of $\mathrm{Ge}$ and $\mathrm{SiO}_{2}$ as an effective material with unknown optical constants modelled by oscillators enables optical characterization of inhomogeneous films. This approach also takes into account the change of optical constants with the size of Ge clusters and to some extent avoids the problem of choosing the proper EMA for the type of the mixture. In the case of inhomogeneous layers, there is a limitation in that the change of optical constants due to the change of cluster size through the layer is not taken into account.

Results obtained from ellipsometric data are in accordance with the results obtained from grazing incidence wide angle $\mathrm{x}$-ray scattering. Ellipsometry has been shown to be an appropriate non-invasive technique for characterization of such layers. Relying on the ellipsometric measurements only, it is possible to find the conditions for the onset of crystallization, distribution of Ge clusters through the layer (via refractive index profile) and to check the trend of growth of crystallites (via $E_{1}$ shift). 


\section{References}

[1] C. Bostedt, T. van Buuren, T. M. Willey, N. Franco, L.J. Terminello, C.Heske, T. Moeller, Appl. Phys. Lett. 84 (2004) 4056.

[2] U.V. Desnica, K. Salamon, M. Buljan, P. Dubček, N. Radić, I.D. Desnica-Franković, Z. Siketić, I. Bogdanović-Radović, M. Ivanda, S. Bernstorff, Superlattice. Microstruct. 44 (2008) 323.

[3] K. Salamon, O. Milat, M. Buljan, U.V. Desnica, N. Radić, P. Dubček, S. Bernstorff, Thin Solid Films 517 (2009) 1899.

[4] M. Buljan, U.V. Desnica, G. Dražić, M. Ivanda, N. Radić, P. Dubček, K. Salamon, S. Bernstorff, V. Holy, Nanotechnology 20 (2009) 085612.

[5] J. Sancho-Parramon, V. Janicki, H. Zorc, Thin Solid Films 516 (2008) 5478.

[6] M. Mansour, A.E. Naciri, L. Johann, S. Duguay, J.J. Grob, M. Stchakovsky, C. Eypert, J. Phys. Chem. Solids 67 (2006) 1291.

[7] B. Gallas, J. Rivory, J. Appl. Phys. 94 (2003) 2248.

[8] M.M. Giangregorio, M. Losurdo, M. Ambrico, P. Capezzuto, G. Bruno, L. Tapfer, J. Appl. Phys. 99 (2006) 063511.

[9] M.I. Alonso, M. Garriga, A. Bernardi, A.R. Goni, A.F. Lopeandia, G. Garcia, J. Rodriguez-Viejo, J.L. Labar, Phys. Status Solidi A 205 (2008) 888.

[10] G. A. Niklasson, C. G. Granqvist, and O. Hunderi, Appl. Opt. 20 (1981) 26.

[11] S. Bosch, J. Ferré-Borrull, N. Leinfellner, A.Canillas, Surf. Sci. 453 (2000) 9.

[12] J.A. Woollam Co., Inc., Guide to Using WVASE32, WexTech Systems, Inc., New York NY, 2008.

[13] A.S. Ferlauto, G.M. Ferreira, J.M. Pearce, C.R. Wronski, R.W. Collins, X.M. Deng G. Ganguly, J. Appl. Phys. 92 (2002) 2424.

[14] M. Yang, T.P. Chen, J.I. Wong, Y. Liu, L. Ding, K.Y. Liu, S. Zhang, W.L. Zhang, D. Gui, C.Y. Ng, J. Phys. D 42 (2009) 035109.

[15] J. Sancho-Parramon, V. Janicki, Journal of Physics D, 41 (2008), 215304.

[15] S.B. White, D.R. McKenzie, Appl.Opt. 27 (1988) 3344.

[16] S. Adachi, Phys. Rev. B 38 (1988) 12966.

[17] S. Adachi, J. Appl. Phys. 66 (1989) 3224. 
[18] M.I. Alonso, M. Garriga, A. Bernardi, A.R. Goni, A.F. Lopeandia, G. Garcia, J. Rodriguez-Viejo, U. Labar, Thin Solid Films 516 (2008) 4277.

[19] B.E. Warren, X-ray diffraction, Addison-Wesley Publishing Company, Reading MA, 1969.

[20] M. Essid, J.L. Brebner, J. Albert, K. Awazu, Nucl. Instrum. Meth. B 141 (1998) 616. 

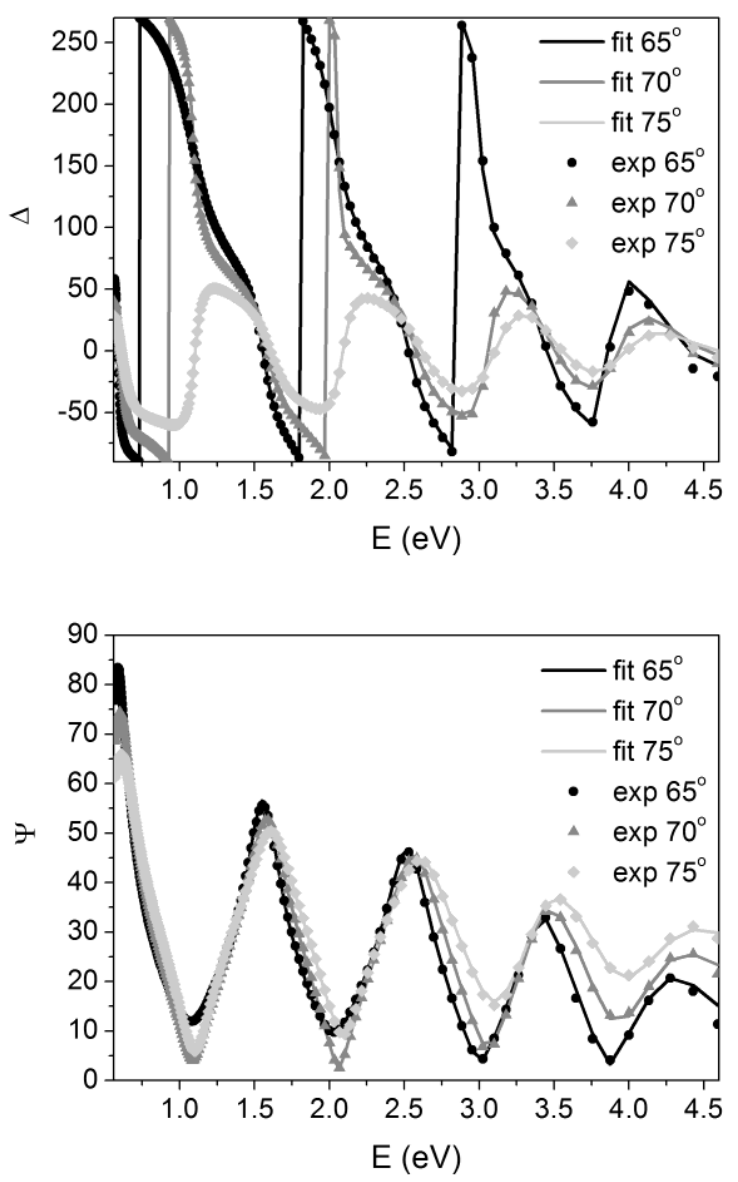

Fig. 1. Data and fit of the sample deposited at substrate heated at $300^{\circ} \mathrm{C}$ and annealed at $600^{\circ} \mathrm{C}$. This sample has fit with the worst final quality. However, it is not possible to distinguish data from the fit by eye. 

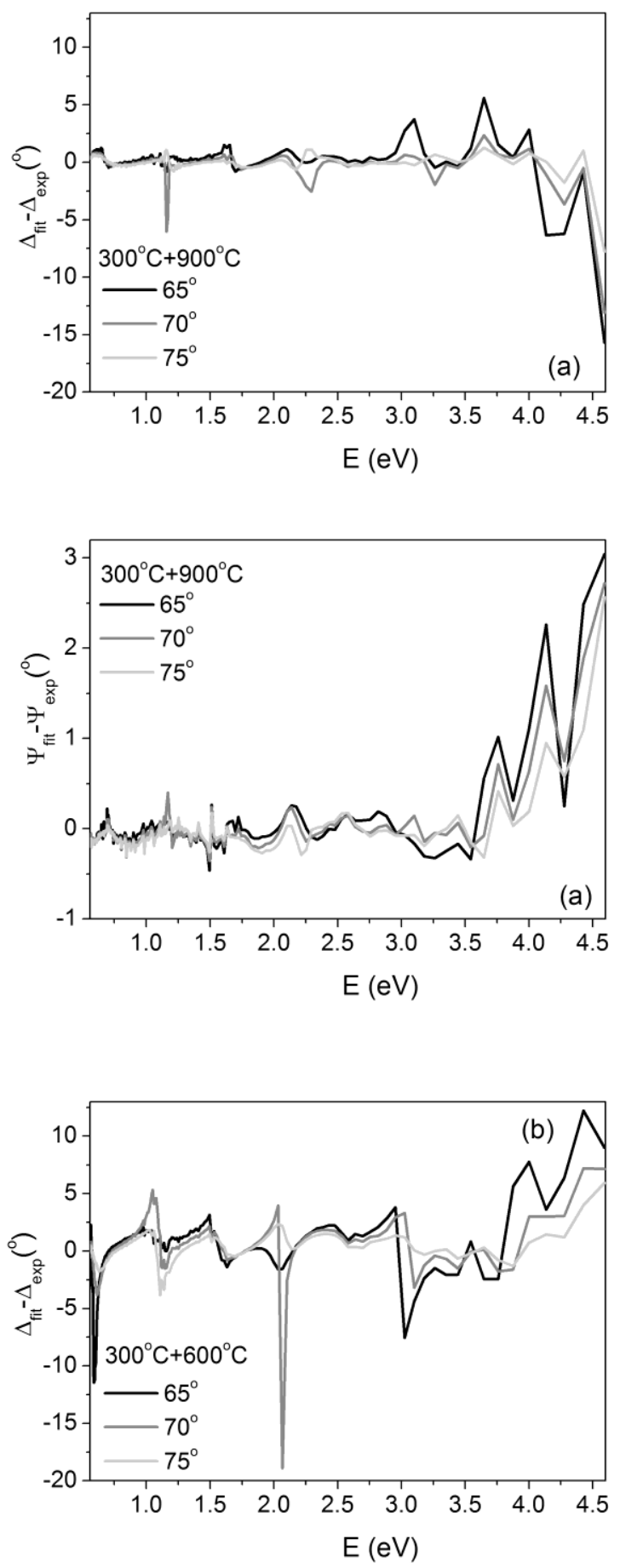


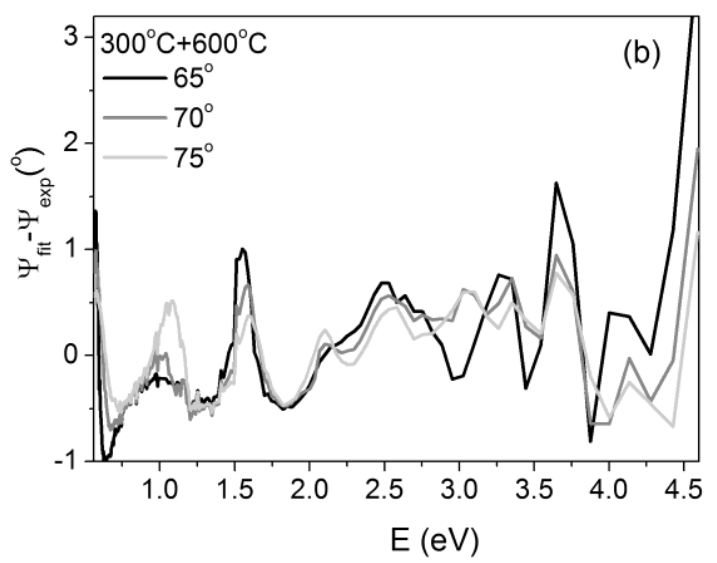

Fig. 2. Difference of fit to the experimental data for the best (a) and the worst (b) fit of the studied samples.

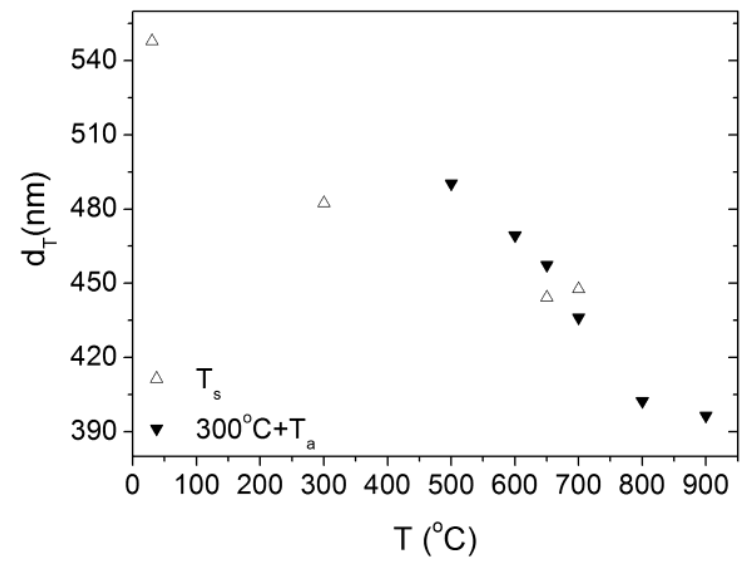

Fig. 3. Total thickness of the layer $\left(d_{T}\right)$ is reducing as $\mathrm{T}_{\mathrm{s}}$ and $\mathrm{T}_{\mathrm{a}}$ are increasing.

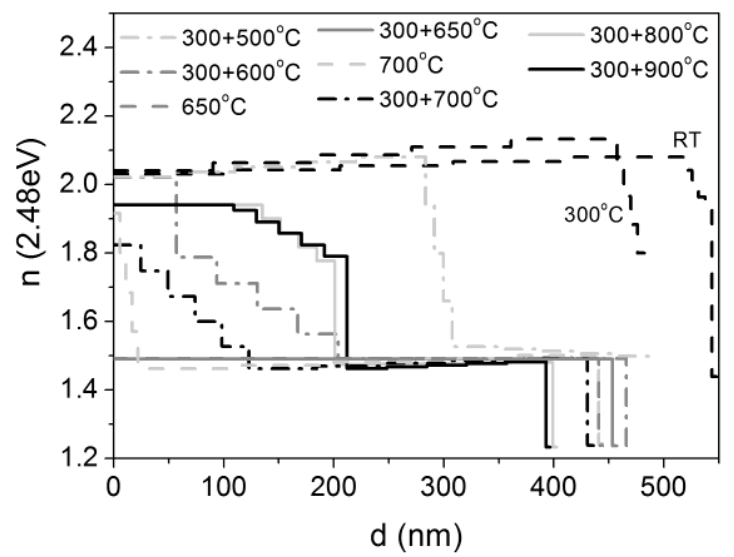

Fig. 4. Change of refractive index profile with $\mathrm{T}_{\mathrm{s}}$ and $\mathrm{T}_{\mathrm{a}}$. 


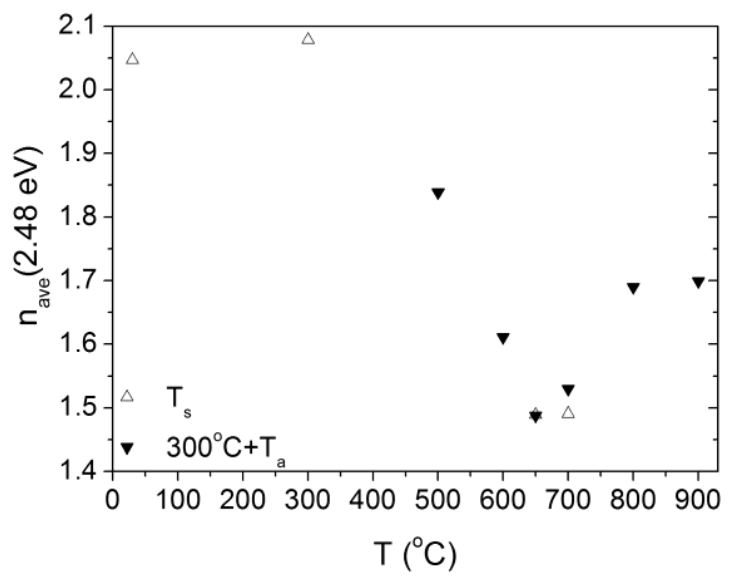

Fig. 5. Change of average refractive index with $\mathrm{T}_{\mathrm{s}}$ and $\mathrm{T}_{\mathrm{a}}$.

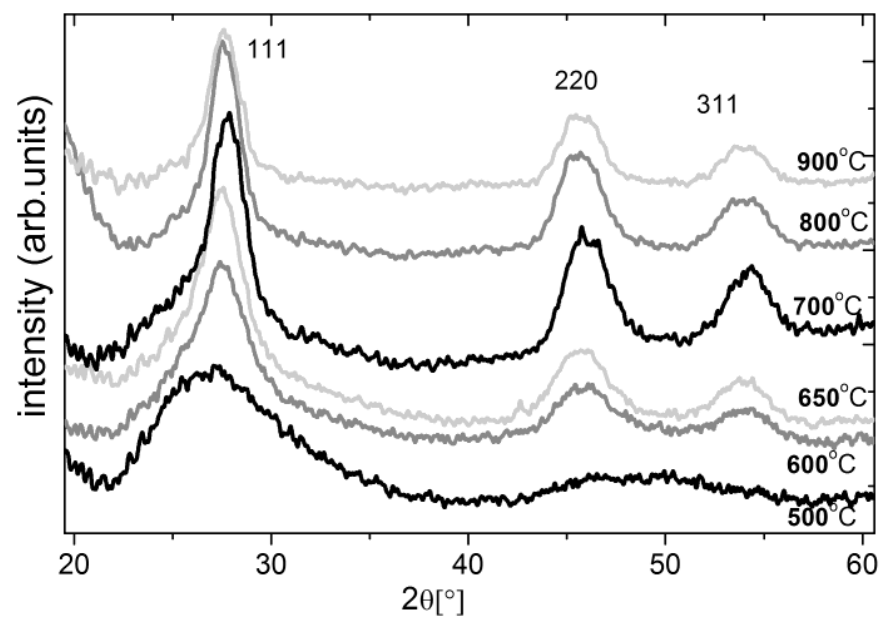

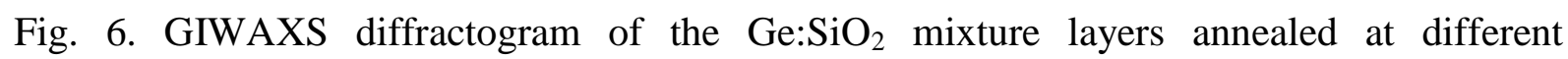
temperatures.

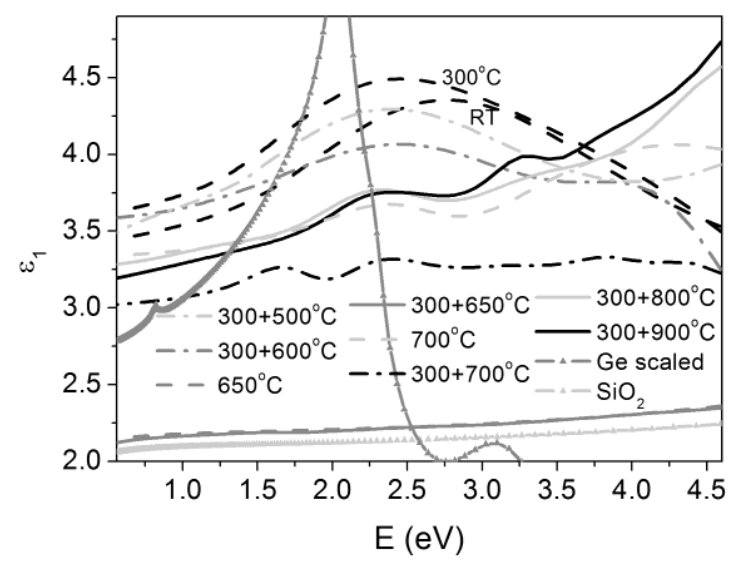

Fig. 7. Change of real part of dielectric function with $\mathrm{T}_{\mathrm{s}}$ and $\mathrm{T}_{\mathrm{a}}$. The real values of $\varepsilon_{\boldsymbol{l}}(\mathbf{G e})$ are 6 times higher. They are scaled here to fit into the graph. 


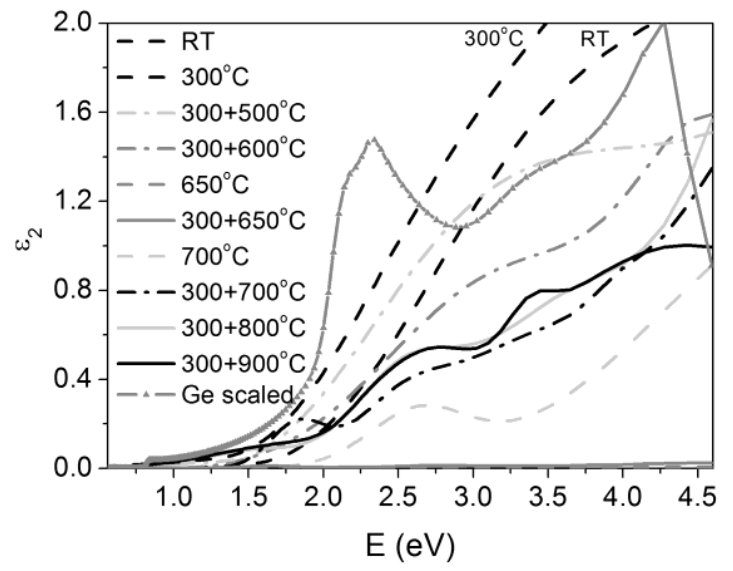

Fig. 8. Change of imaginary part of dielectric function with $T_{s}$ and $T_{a}$. The real values of $\varepsilon_{2}(\mathrm{Ge})$ are 16 times higher. They are scaled here to fit into the graph.

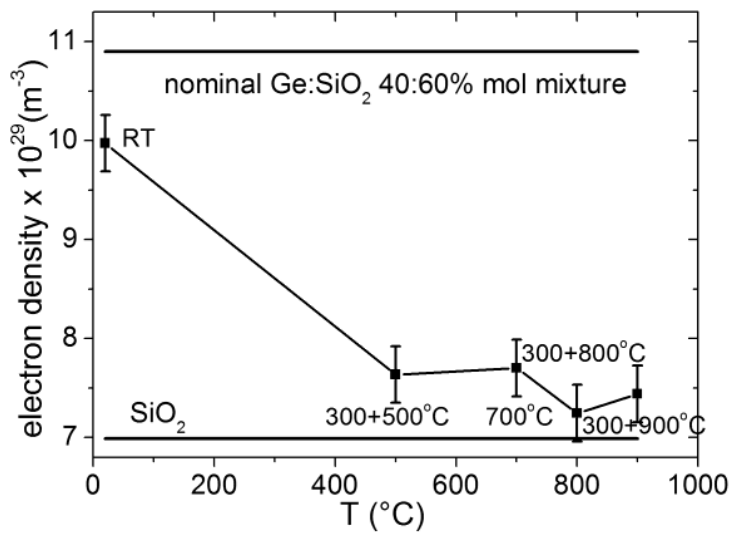

Fig. 9. Dependence of electron density in the surface of the samples with temperature of substrate or annealing.

\section{List of table captions}

Table 1. Models used for optical constants of material $M$ and the position of $E_{1}$ peak. Energy $E_{1}$ for bulk Ge is $2.1 \mathrm{eV}$. 
Tables

Table 1

\begin{tabular}{|c|c|c|}
\hline Ts/T & model & $\mathrm{E}_{1}(\mathrm{eV})$ \\
\hline $\mathrm{RT} /-$ & Gauss+CL & \\
\hline $300 /-$ & Gauss+CL & \\
\hline $300 / 500$ & Gauss+CL & \\
\hline $300 / 600$ & Gauss+CL & 2.36 \\
\hline $650 /-$ & Cauchy & \\
\hline $300 / 650$ & 4 Gauss oscillators & \\
\hline $700 /-$ & 2 Gauss oscillators & 2.34 \\
\hline $300 / 700$ & 5 Gauss oscillators & 2.36 \\
\hline $300 / 800$ & 4 Gauss oscillators & 2.19 \\
\hline $300 / 900$ & 5 Gauss oscillators & 2.19 \\
\hline
\end{tabular}

